\title{
Effect of COVID-19 on Orthopedic Medical Education in Tamil Nadu
}

Coronavirus disease-2019 (COVID-19) is a real threat to mankind. The activites of daily living have been completely paralyzed due to this pandemic.

\section{Undergraduate Medical Education}

Regarding undergraduate medical education, the usual clinical demonstration classes got suspended due to pandemic restrictions. Theory classes which were generally conducted in lecture halls, were converted into online mode classes. Clinical postings in orthopedics for the undergraduates were suspended. Final year MBBS university clinical examination was conducted as case study without examining the patients. I am afraid that this type of education during the pandemic may lower the level of undergraduate medical education.

\section{Postgraduate Medical Education}

Orthopedic postgraduates were diverted to COVID activities. Hence the postgraduate training in orthopedics was severely affected due to COVID activities. This is happening for more than a year since March 2020. During the COVID-19 lockdown, the number of fracture patients coming to the hospital have reduced. More number of elective orthopedic theaters were also closed affecting the surgical training in non-traumatic orthopedics for the postgraduates. The hands-on training in surgical skill development in orthopedics for the postgraduates have been affected in COVID-19 pandemic. Even the number of patients coming to the outpatient department were also decreased leading to reduced clinical exposure to orthopedic postgraduates. Even the postgraduate theory classes were conducted in online mode and were utilized to maximum extent. Thus, this COVID-19 pandemic has shifted the means of orthopedic medical education from offline to online mode.

The Tamilnadu Orthopedic Association have conducted clinical examination series for the postgraduates utilizing the online platform.

The Association have also conducted Clinical examination Course for the postgraduates in offline mode and helped the postgraduates to update their clinical skill.

Usually Various CMEs and cadaveric workshops were conducted with credit points from the University. All such academic sessions are stopped during COVID-19 pandemic. With the absence of such academic sessions and workshops, postgraduates have been denied the opportunity and chance to interact and learn the surgical skill and updating orthopedic knowledge during COVID-19 pandemic in Tamil Nadu.

Moreover, the residents are mentally stressed regarding their academic training and academic examinations apart from psychological stress while working in COVID ward activities.

Singla et al. have stated that the surgical exposure, hands-on training and academic training of the orthopedic residents in various North Indian Institutions have been affected along with burden of mental stress related to COVID duties. ${ }^{1}$ Upadhyaya et al. reported that the orthopedic residents in Delhi and its surrounding regions had significant reduction in their theory classes, clinical and practical training and inability to continue their research activities. ${ }^{2}$ According to Mukherjee K et al., there was an undeniable psychological impact on the postgraduates apart from the reduced surgical exposure and training among orthopedic residents in south India. ${ }^{3}$ According to Kumar GS et al., orthopedic residents in India are not getting adequate surgical exposure with some of them getting negligible hands-on surgical training due to the COVID-19 pandemic. ${ }^{4}$

Such impact on the orthopedic education is not limited to Tamil Nadu or India alone but is being experienced world-wide. As an evidence to it, Monica Kogan MD et al. had stated that in the United States of America, the surgical training for the orthopedic residents was affected. ${ }^{5}$ According to Planter et al., the delivery of education and skills training for the orthopedic residents in North America has been critically affected, as a compensation for the impairment in routine training, virtual meeting platforms and simulation-based training are being implemented. ${ }^{6}$ Megaloikonomos et al. have also reported that the orthopedic and trauma training in Europe was significantly affected due to the COVID-19 pandemic.?

Even though Tamil Nadu have the best medical facilities and infrastructure on par with international standards, COVID-19 pandemic have not spared the orthopedic medical education in Tamil Nadu.

S Vetrivel Chezian

\section{References}

1. Singla, M., Kaushal, A., Chopra, R., \& Vermani, S. (2021). The Effect of COVID-19 on Orthopedic Residents' Education, Skills and Mental Health: A Resident's Perspective. Asian Journal of Orthopedic Research, 5(2), 7-21.

2. Gaurav Kumar Upadhyaya, Vijay Kumar Jain, Karthikeyan P. Iyengar, Mohit Kumar Patralekh, Abhishek Vaish, Impact of COVID-19 on post-graduate orthopedic training in Delhi-NCR,Journal of Clinical Orthopedics and Trauma,Volume 11, Supplement 5, 2020.

3. Mukherjee K, Basak I, Prasanth T. Impact of COVID-19 on orthopedic residents in South India: a survey. Int J Community Med Public Health 2021;8:1956-61.

4. Kumar GS, Prasanna MS ORTHOa, Yadav, Amit Kumar MS ORTHOa; Harsoor, Abhishek MS ORTHOa; H, Kantharaju MS ORTHOb; Mane, Akash V MS ORTHOa; Palange, Nikhil D. MS ORTHOc Impact of COVID-19 on orthopedic residents-an Indian perspective, OTA International: June 2021 Volume 4 - Issue 2 - p e096. 
5. Kogan, Monica MD; Klein, Sandra E. MD; Hannon, Charles P. MD, MBA; Nolte, Michael T. MD Orthopedic Education During the COVID-19 Pandemic, Journal of the American Academy of Orthopedic Surgeons: June 1, 2020 - Volume 28 - Issue 11 - p e456-e464

6. Kevin D. Plancher, Jaya Prasad Shanmugam, Stephanie C. Petterson. The Changing Face of Orthopedic Education: Searching for the New Reality After COVID-19,Arthroscopy, Sports Medicine, and Rehabilitation,Volume 2, Issue 4, 2020.

7. Megaloikonomos, P.D., Thaler, M., Igoumenou, V.G. et al. Impact of the COVID-19 pandemic on orthopedic and trauma surgery training in Europe. International Orthopedics (SICOT) 44, 1611-1619 (2020). 